\title{
Jacobsen/Paris-Trousseau Syndrome: Report of a Case with Emphasis on Platelet's Light Microscopic and Ultrastructure Findings
}

\author{
Michael Losos', Basil M. Kahwash2, Miriam Conces³, Joel Thompson', Riten Kumar", \\ Samir B. Kahwash ${ }^{3}$ \\ ${ }^{1}$ Department of Pathology, The Ohio State University Wexner Medical Center, Columbus, $\mathrm{OH}, \mathrm{USA}$ \\ ${ }^{2}$ Indiana University, Indianapolis, IN, USA \\ ${ }^{3}$ Department of Pathology and Laboratory Medicine, Nationwide Children's Hospital, Columbus, OH, USA \\ ${ }^{4}$ Department of Pediatrics, The Ohio State University, Division of Hematology/Oncology/BMT, Nationwide \\ Children's Hospital, Columbus, OH, USA \\ Email: samir.kahwash@nationwidechildrens.org
}

Received 9 November 2015; accepted 1 January 2016; published 6 January 2016

Copyright (C) 2016 by authors and Scientific Research Publishing Inc.

This work is licensed under the Creative Commons Attribution International License (CC BY).

http://creativecommons.org/licenses/by/4.0/

(c) (i) Open Access

\begin{abstract}
Jacobsen syndrome (JS) is a rare, inherited disorder, characterized by facial and skull dysmorphism, mental retardation, and platelet abnormalities. Paris-Trousseau syndrome (PTS) is a platelet function disorder that may be encountered in patients affected by JS. PTS is manifested by a mild lifelong bleeding tendency. Morphologically, the presence of large fused platelet alpha granules is characteristic. We present a case of Jacobsen syndrome in a child and highlight the typical morphologic and ultrastructure findings of platelets.
\end{abstract}

\section{Keywords}

Jacobsen Syndrome, Paris-Trousseau Syndrome, Platelet Microscopic Findings, Electron Microscopy

\section{Introduction}

Jacobsen syndrome (JS) is a very rare disorder that is caused by a congenital partial deletion of chromosome "Corresponding author.

How to cite this paper: Losos, M., Kahwash, B.M., Conces, M., Thompson, J., Kumar, R. and Kahwash, S.B. (2016) Jacobsen/ Paris-Trousseau Syndrome: Report of a Case with Emphasis on Platelet's Light Microscopic and Ultrastructure Findings. Open Journal of Pathology, 5, 8-13. http://dx.doi.org/10.4236/ojpathology.2016.61002 
11q23. The estimated incidence is 1 in 100,000 newborns, and the female to male ratio is 2:1 [1]. To date, over 200 people have been identified as being affected with JS. The disease is characterized by facial dysmorphism with features including hypertelorism, ptosis, epicanthal folds, small and low-set ears, macrocephaly, thin upper lip, broad nasal bridge, downturned corners of the mouth, and small mandible. Affected individuals often have trigonocephaly a skull abnormality creating the impression of a pointed forehead [2]-[4]. Multiple organ systems are affected. Common features include feeding difficulties in infancy, congenital heart defects, genitourinary abnormalities, short stature, skeletal abnormalities, and frequent sinus and ear infections. The central nervous system is also affected, which may lead to learning disabilities, impaired speech, delayed motor skills, sensory problems, and behavioral concerns [1]-[3]. Clinical outcome is variable, and approximately $20 \%$ of children die within the first 2 years of life [5]. Congenital heart disease is the most common cause of death in children with JS. The life expectance of patients who survive to adulthood is unknown. Given the rarity of JS, a better understanding of its clinical course will require long-term follow-up and collaborative studies [2] [5].

Approximately $90 \%$ of subjects with JS have a mild bleeding disorder called Paris-Trousseau syndrome (PTS), a macrothrombocytopenia which results in muco-cutaneous bleeding symptoms [2] [3]. Many of the patients diagnosed with PTS present with thrombocytopenia/pancytopenia, which may improve later in life. However, the increase in platelet count does not compensate for the associated platelet dysfunction [2]-[4] [6]. Approximately $15 \%$ of platelets in subjects with PTS exhibit large, fused alpha granules that do not release their contents during coagulation. As a result, the platelets exhibit a diminished response to thrombin and result in an increased risk of bleeding in affected individuals [3].

Our focus is on the platelet pathology that is present on the peripheral blood smear. We describe the characteristic light microscopic morphologic features of the abnormal platelets, as well as provide useful photomicrographs that can serve as diagnostic reference.

\section{Case Presentation}

The patient, a 4-year-old male, was initially referred to our institution after receiving a diagnosis of Jacobsen syndrome (JS) two weeks after birth. By cytogenetics, the patient had an unbalanced translocation with partial monosomy of the long arm of chromosome 11 and partial trisomy of the long arm of chromosome 16 . The partial deletion of $11 \mathrm{q}$ involved the region associated with JS. The father was a known carrier of a balanced translocation prior to the patient's birth. Physical exam at birth included nasoseptal deviation, hypospadias, and unilateral undescended testicle. Echocardiogram revealed a ventricular septal defect, and laboratory evaluation demonstrated moderate thrombocytopenia which ranged from 49,000 to 55,000 during the first week of life. Seizures began at 4 months of age and responded well to Topamax.

The patient's platelet count at birth was $52 \mathrm{~K} / \mathrm{cu} \mathrm{mm}$. Due to expected platelet dysfunction, all surgeries and procedures were preceded by prophylactic platelet transfusions. Early surgical history included nasal septum repair, performed at 10 weeks of age, and multiple urological procedures. His complete blood count (CBC) was followed regularly, and in the first year of life, the platelet count ranged from $32 \mathrm{~K} / \mathrm{cu} \mathrm{mm}$ to $125 \mathrm{~K} / \mathrm{cu} \mathrm{mm}$. By 4 years of age, his thrombocytopenia had resolved.

At the age of 4, additional platelet studies were performed during a pre-operative evaluation for additional urologic procedures. Work up at this time included a CBC, peripheral blood smear review by a pathologist, electron microscopy (EM), and platelet aggregation studies. His platelet count was $155 \mathrm{~K} / \mathrm{cu} \mathrm{mm}$ (Table 1). Upon examination, his peripheral blood smear was significant for large platelets, some showing prominent clumped red granules (Figure 1 and Figure 2), raising the possibility of Jacobsen/Paris Trousseau syndrome (JPT) [2] [3]. Peripheral blood platelet aggregation studies revealed diminished aggregation with low-dose epinephrine and increased lag time with collagen. EM of the peripheral blood platelets showed an average of 2.08 delta granules per platelet, with the presence of large clumped alpha granules (Figure 3), as would be expected in JPT [7].

\section{Discussion}

Jacobsen syndrome (JS) is a rare disorder due to variable-sized deletions in the long arm of chromosome 11, characterized by facial dysmorphism, growth retardation, skeletal abnormalities, and multiple systemic dysfunctions, including hematologic abnormalities [5]. Due to the complex nature of JS and the presence of abnormalities involving multiple organ systems, care for these patients requires a multidisciplinary team approach of pe- 


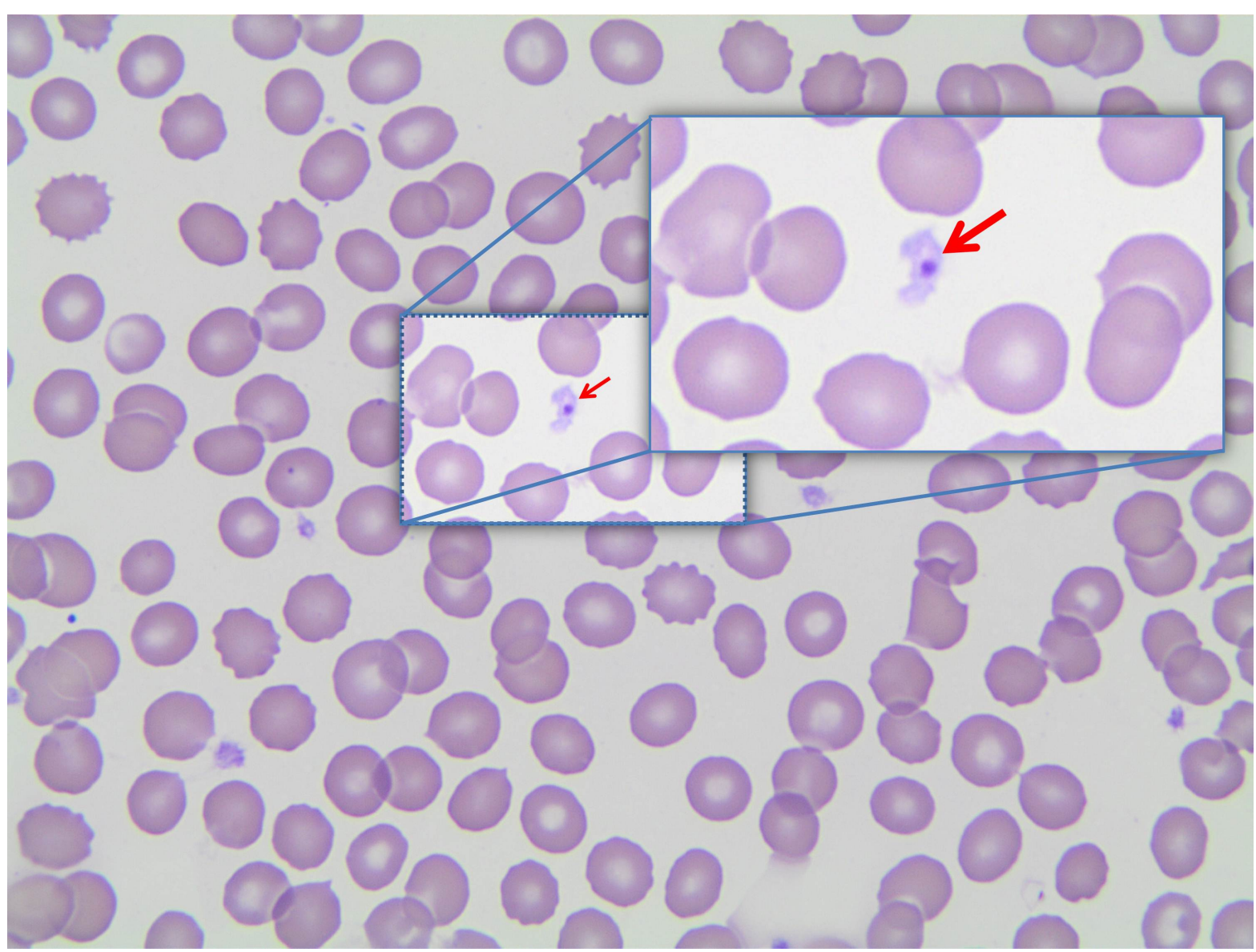

Figure 1. Photomicrograph from patient's peripheral blood smear demonstrating an abnormal platelet with fusion of alpha granules into one large clumped granule (Arrow). Magnification is $100 \times$ oil.

Table 1. Complete blood count results.

\begin{tabular}{ccc}
\hline & Ranges of patient’s results during follow up & Normal ranges \\
\hline Red blood cells & $3.16-4.71 \mathrm{M} / \mathrm{cu} \mathrm{mm}$ & $3.9-5.3 \mathrm{M} / \mathrm{cu} \mathrm{mm}$ \\
Hemoglobin & $9.2-16.7 \mathrm{~g} / \mathrm{dL}$ & $11.5-13.5 \mathrm{~g} / \mathrm{dL}$ \\
Hematocrit & $27.3 \%-48 \%$ & $34 \%-40 \%$ \\
Mean corpuscular volume & $74.8-104.2 \mathrm{fL}$ & $75-88 \mathrm{fL}$ \\
Mean corpuscular hemoglobin concentration & $32.1 \%-35.5 \%$ & $31.0 \%-37.0 \%$ \\
Red blood cell distribution width & $13.6 \%-19.3 \%$ & $10 \%-14.1 \%$ \\
White blood cells & $7-14.1 \mathrm{~K} / \mathrm{cu} \mathrm{mm}$ & $5.5-15.5 \mathrm{~K} / \mathrm{cu} \mathrm{mm}$ \\
Platelets & $32-155 \mathrm{~K} / \mathrm{cu} \mathrm{mm}$ & $140-440 \mathrm{~K} / \mathrm{cu} \mathrm{mm}$ \\
Mean platelet volume & $10.4-12.7 \mathrm{fL}$ & $9.3-13.0 \mathrm{fL}$ \\
\hline
\end{tabular}

diatricians, pediatric cardiologists, hematologists, neurologists, and other specialists.

The facial dysmorphisms of JS are distinctive. However, this disorder shows a wide spectrum of physical features, with some clinical features overlapping with Turner syndrome and Noonan syndrome. The latter include: short (possibly webbed) neck, short stature, down slanting palpebral fissures, facial dysmorphism, ptosis, 


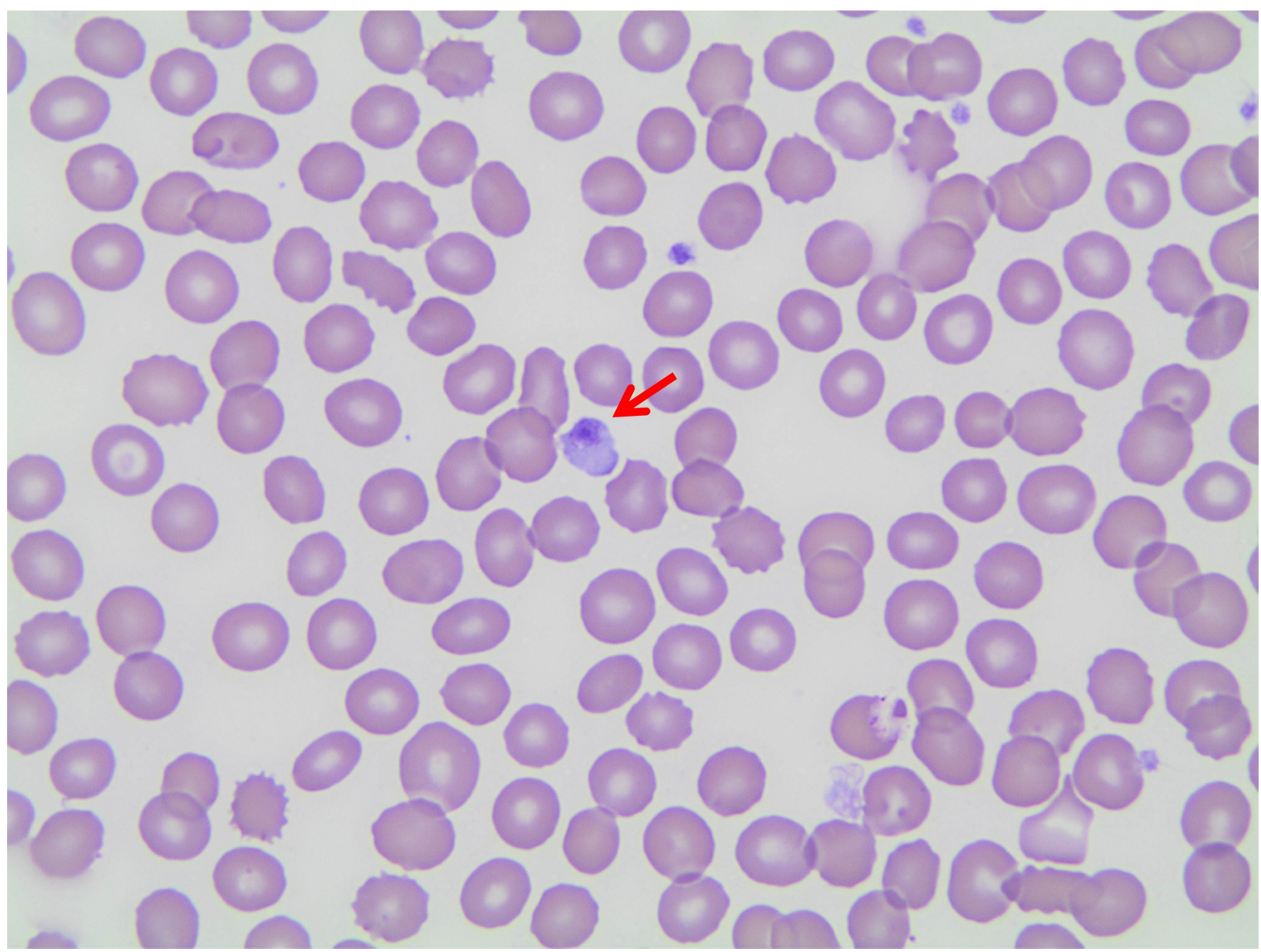

Figure 2. Peripheral blood smear with a large atypical platelet (center) showing alpha granules merged into one huge clump. Magnification is $100 \times$ oil.

and aortic or pulmonary stenosis [5]. As in patients with JS, children with Noonan syndrome may also present with thrombocytopenia and an increased risk of bleeding.

Most patients with JS are born with thrombocytopenia and some may even have pancytopenia; which often resolves over the first few years of life [5]. Additionally, the bone marrow demonstrates dysmegakaryopoesis with an increase in the number of small immature platelets [8].

From a pathologic standpoint, microscopic examination of the peripheral blood smear demonstrates distinct morphologic abnormalities including thrombocytopenia and large platelets with prominent red alpha granules. These findings can be the initial clues to the diagnosis of JPT [3]. Platelet aggregation studies may show abnormalities, and provide further evidence of platelet dysfunction. EM is useful in evaluation of the dense and alpha granules in PTS. Alpha granules may fuse to form spherical or irregular giant granules, and these typically are identified in approximately 15\% the platelets [6] [7]. These ultrastructural findings help in confirming the diagnosis, considering that alpha granules in normal platelets are relatively uniform in size. Interpretation of platelet EM requires awareness that occasional large alpha granules may be seen as an artifact in blood specimens after prolonged storage [7]. Other pathologic findings in platelets of patients with JS include a decreased number of platelets dense or delta granules [7]. The platelet abnormalities seen in PTS result in poor platelet function and increased risk of bleeding [1]-[3].

Our patient had one complication of significant bleeding post-operatively; epistaxis post nasal septum repair. For subsequent surgeries, including complex urological procedures, he received pre-operative platelets and did not develop any hemorrhagic symptoms. Although his thrombocytopenia resolved, his platelet aggregation studies revealed diminished function, and platelet ultrastructure revealed the typical structural abnormalities of JPT. The patient likely has a mild phenotype of a syndrome known for variable penetrance [5]. Prevention of bleeding symptoms in patients with JPT is multi-disciplinary and includes lifestyle modifications, including avoid- 


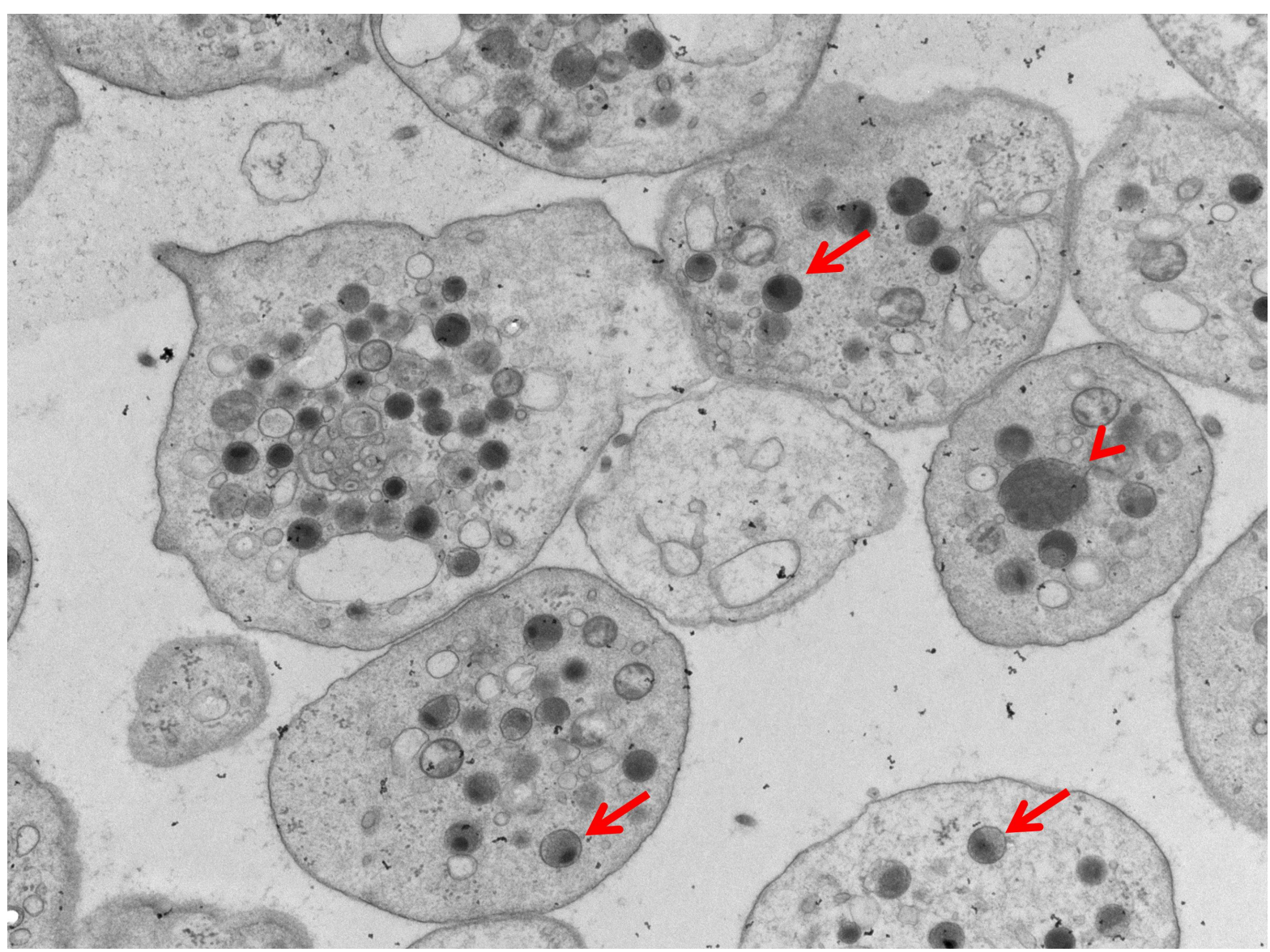

Figure 3. Transmission Electron Microscopic photo of large abnormal platelets in Jacobsen syndrome demonstrating large (arrows) alpha granules. A giant fused alpha granule is noted (Arrow head). Magnification is 15,000×.

ance of contact and collision sports, routine dental care to reduce the risk of gingival bleeding, avoidance of platelet impairing medications (aspirin and nonsteroidal anti-inflammatory drugs), and use of medic alert bracelets. Mild-to-moderate bleeding episodes may be managed with local measures; such as compression, nasal packing (for epistaxis), and topical human thrombin. Specific regimens, including anti-fibrinolytic medications (lysine analogs), may be used alone or in combination with local measures. Platelet transfusions are usually reserved for life threatening bleeds or may be used pre-operatively [9].

While the facial dysmorphism and platelet abnormalities are distinctive clues to JS, cytogenetic analysis is necessary for confirmation. Cytogenetic analysis consistently demonstrates a partial deletion of the long arm of chromosome 11q23. The size of the deletion varies significantly, and severe clinical features are seen with larger deletions. The majority of the deletions are a result of de novo mutations [5]. Furthermore, incomplete penetrance appears to contribute to widening of the spectrum of clinical phenotype. Of note is that the FLI-1 gene has been identified in the $11 \mathrm{q}$ region, with evidence that certain proteins coded by this gene may play a role in megakaryocyte differentiation [8].

\section{Conclusion}

Jacobsen/Paris Trousseau syndrome is a rare platelet function disorder with characteristically large platelet alpha granules that can be easily appreciated upon careful review of a well stained peripheral blood smear. The finding is fairly specific on light and electron microscopic examination, especially if an artifactual change resulting from prolonged storage of blood is excluded. Despite these facts, there is scant literature and no quality images available on the internet demonstrating these morphologic platelet findings in this disorder. The publication of an illustrated case report, such as this, may help compensate for this void and provide practicing physicians and students a much needed, readily accessible resource. 


\section{Acknowledgements}

The authors thank Maria Nunez for her help in preparing this manuscript.

\section{References}

[1] Breton-Gorius, J., Favier, R., Guichard, J., Cherif, D., Berger, R., et al. (1995) A New Congenital Dysmegakaryopoietic Thrombocytopenia (Paris-Trousseau) Associated with Giant Platelet Alpha-Granules and Chromosome 11 Deletions at 11q23. Blood, 85, 1805-1814.

[2] Jacobsen, P., Hauge, M., Henningsen, K., Hobolth, N., Mikkelsen, M. and Philip, J. (1973) An (11;21) Translocation in Four Generations with Chromosome 11 Abnormalities in the Offspring. A Clinical, Cytogenetical, and Gene Marker Study. Human Heredity, 23, 568-585. http://dx.doi.org/10.1159/000152624

[3] Favier, R., Jondeau, K., Boutard, P., Grossfeld, P., Reinert, P., Jones, C., et al. (2003) Paris-Trousseau Syndrome: Clinical, Hematological, Molecular Data of Ten New Cases. Thrombosis and Haemostasis, 90, 893-897. http://dx.doi.org/10.1160/TH03-02-0120

[4] Wenger, S.L., Grossfeld, P.D., Siu, B.L., Coad, J.E., Keller, F.G. and Hummel, M. (2006) Molecular Characterization of an 11q Interstitial Deletion in a Patient with the Clinical Features of Jacobsen Syndrome. American Journal of Medical Genetics Part A, 140A, 704-708. http://dx.doi.org/10.1002/ajmg.a.31146

[5] Mattina, T., Perrotta, C. and Grossfeld, P. (2009) Jacobsen Syndrome. Orphanet Journal of Rare Diseases, $4,9$. http://dx.doi.org/10.1186/1750-1172-4-9

[6] Krishnamurti, L., Neglia, J.P., Nagarajan, R., Berry, S.A., Lohr, J., Hirsch, B. and White, J.G. (2001) Paris-Trousseau Syndrome Platelets in a Child with Jacobsen’s Syndrome. American Journal of Hematology, 66, 295-299. http://dx.doi.org/10.1002/ajh.1061

[7] White, J.G. (2007) Platelet Storage Pool Deficiency in Jacobsen Syndrome. Platelets, 18, 522-527. http://dx.doi.org/10.1080/09537100701280670

[8] Favier, R., Akshoomoff, N., Mattson, S. and Grossfeld, P. (2015) Jacobsen Syndrome: Advances in Our Knowledge of Phenotype and Genotype. American Journal of Medical Genetics Part C: Seminars in Medical Genetics, 169, $239-250$. http://dx.doi.org/10.1002/ajmg.c.31448

[9] Kumar, R. and Kahr, W. (2013) Congenital Thrombocytopenia: Clinical Manifestations, Laboratory Abnormalities, and Molecular Defects of a Heterogeneous Group of Conditions. Hematology/Oncology Clinics of North America, 27, 465-494. http://dx.doi.org/10.1016/j.hoc.2013.02.004 